This item was submitted to Loughborough's Research Repository by the author.

Items in Figshare are protected by copyright, with all rights reserved, unless otherwise indicated.

\title{
Polynomial matrix QR decomposition and iterative decoding of frequency selective MIMO channels
}

PLEASE CITE THE PUBLISHED VERSION

PUBLISHER

(c) IEEE

VERSION

VoR (Version of Record)

\section{LICENCE}

CC BY-NC-ND 4.0

\section{REPOSITORY RECORD}

Davies, Martin, Sangarapillai Lambotharan, Joanne Foster, Jonathon Chambers, and John McWhirter. 2019. "Polynomial Matrix QR Decomposition and Iterative Decoding of Frequency Selective MIMO Channels". figshare. https://hdl.handle.net/2134/5536. 
This item was submitted to Loughborough's Institutional Repository (https://dspace.lboro.ac.uk/) by the author and is made available under the following Creative Commons Licence conditions.

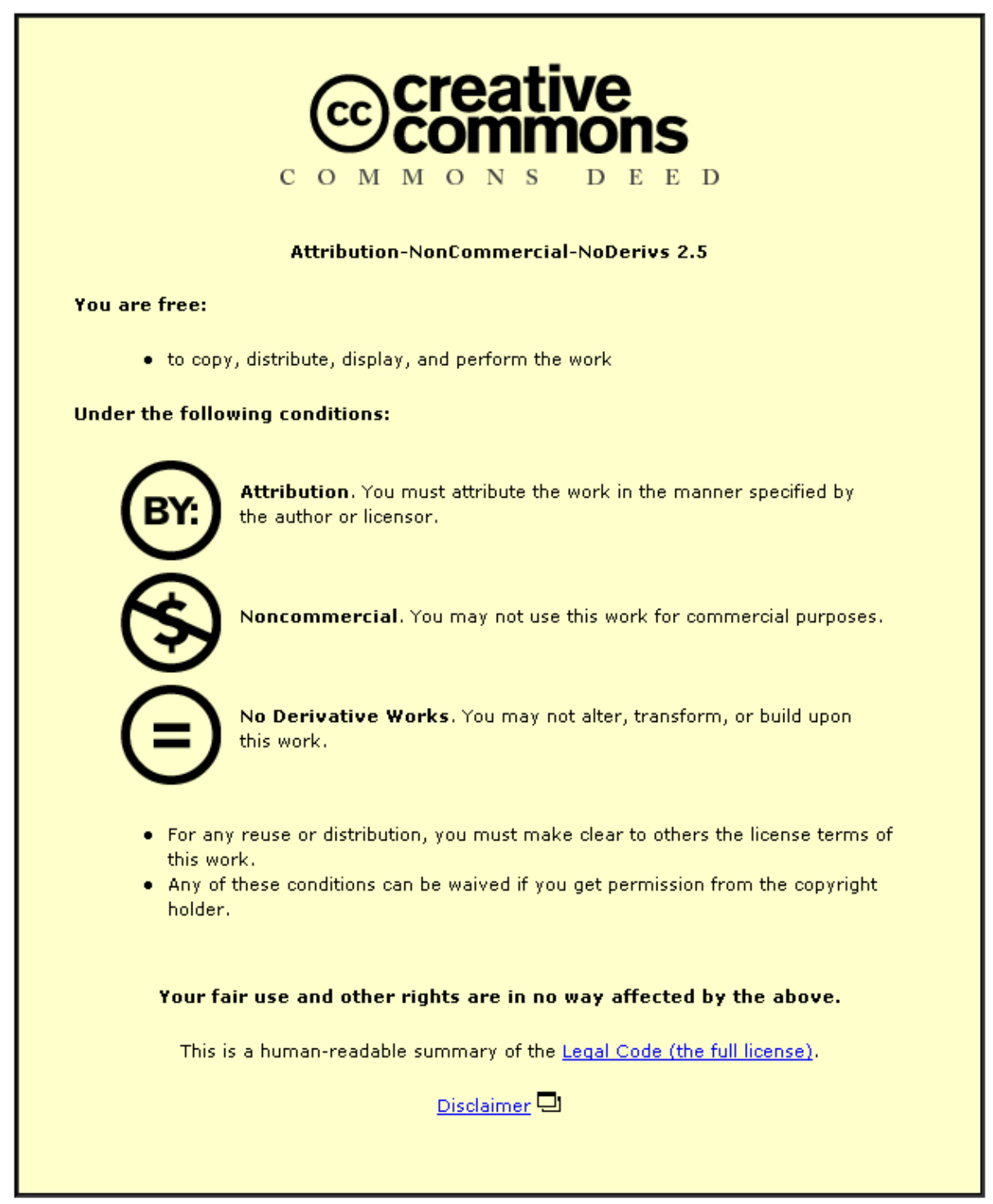

For the full text of this licence, please go to: http://creativecommons.org/licenses/by-nc-nd/2.5/ 


\section{Polynomial Matrix QR Decomposition and Iterative Decoding of Frequency Selective MIMO Channels}

\author{
Martin Davies, Sangarapillai Lambotharan \\ Joanne Foster and Jonathon Chambers \\ Advanced Signal Processing Group \\ Department of Electronic and Electrical Engineering \\ Loughborough University LE11 3TU, UK \\ Email: M.R.Davies, S.Lambotharan, J.A.Foster, J.A.Chambers@lboro.ac.uk
}

\author{
John McWhirter \\ Center for D.S.P. \\ School of Engineering \\ Cardiff University CF24 3AA, UK \\ Email: McWhirterJG@cardiff.ac.uk
}

\begin{abstract}
For a frequency flat multi-input multi-output (MIMO) system the QR decomposition can be applied to reduce the MIMO channel equalization problem to a set of decision feedback based single channel problems. Using a novel technique for polynomial matrix QR decomposition (PMQRD) based on Givens rotations, we show the PMQRD can do likewise for a frequency selective MIMO system. Two types of transmitter design, based on Horizontal and Vertical Bell Laboratories Layered Space Time (H-BLAST, V-BLAST) encoding have been implemented. Receiver processing utilizes Turbo equalization to exploit multipath delay spread and to facilitate multi-stream data feedback.

Average bit error rate simulations show a considerable improvement over a benchmark orthogonal frequency division multiplexing (OFDM) technique. The proposed scheme thereby has potential applicability in MIMO communication applications, particularly for a TDMA system with frequency selective channels.
\end{abstract}

\section{INTRODUCTION}

For a wireless system consisting of $M_{t}$ transmit antennas and $M_{r}$ receive antennas, the noise free channel can be represented as an $M_{r} \times M_{t}$ matrix, $\boldsymbol{H}$. In a frequency flat scenario where the received signals are instantaneously mixed, a scalar matrix is sufficient to describe the mixing. If the channel matrix is known to the receiver, its $\mathrm{QR}$ decomposition [1] can be formulated. Decomposing the channel matrix and exploiting the upper triangular structure of the resulting matrix, the set of source signals can be retrieved from the received signals using back substitution [2]. In the case of a frequency selective wireless system, the noise free multipath channel can be represented by a polynomial matrix, $\underline{\boldsymbol{H}}(z)$

$$
\underline{\boldsymbol{H}}(z)=\sum_{i=0}^{L-1} \boldsymbol{H}_{i} z^{-i}
$$

where $\boldsymbol{H}_{i} \in \mathbb{C}^{M_{r} \times M_{t}}$ is the $i^{t h}$ matrix tap of the MIMO channel of length $L$ and $z^{-i}$ is the unit delay operator. Thus its QR decomposition cannot be directly formulated. A typical approach to this broadband problem is to reduce it to a narrowband form by using a discrete Fourier transform (DFT) to split the broadband channel into narrow uniformly spaced frequency bands and applying the QR decomposition in each band. This is suitable for an OFDM based system. However in this paper, we propose a direct time domain based polynomial matrix decomposition as this is applicable to a range of access schemes such as time division multiple access (TDMA).

\section{A. Choice of Notation}

Throughout this paper, matrices are denoted by upper case bold characters and vectors by lower case bold characters. Regular upper or lower case characters denote scalar quantities. $[\cdot]_{k l}$ denotes the $(k, l)$-th element of the matrix in the square brackets. The superscripts $*, T$, and $H$ denote the complex conjugate, matrix transpose and Hermitian conjugate, respectively. $\boldsymbol{I}_{p}$ is used to denote the $p \times p$ identity matrix. Polynomial matrices and vectors are denoted by underscored bold upper and lower case characters, respectively. The use of an underscore with scalar quantities denotes a polynomial with scalar coefficients. Any polynomial (matrix, vector, or scalar) with the qualifier $(z)$ denotes a polynomial in the indeterminate variable $z^{-1}$. The $*$, used as a subscript, denotes complex conjugation of the coefficients in a polynomial matrix or vector. The use of $\sim$ above a polynomial matrix or vector denotes the paraconjugate, i.e. for a given polynomial matrix $\underline{\boldsymbol{A}}(z), \underline{\widetilde{\boldsymbol{A}}}(z)=\underline{\boldsymbol{A}}_{*}^{T}\left(z^{-1}\right) .\|\cdot\|_{F}$ will be used to denote the Frobenius norm (F-norm) of a polynomial matrix, which is simply the square root of sum of the squared F-norms for all coefficient matrices.

\section{QR DECOMPOSITION}

The $\mathrm{QR}$ decomposition of a $p \times q$ complex scalar matrix, $\boldsymbol{A}$ is given as:

$$
\boldsymbol{A}=\boldsymbol{Q R}
$$

where $\boldsymbol{Q}$ is a $p \times p$ unitary matrix, so that $\boldsymbol{Q} \boldsymbol{Q}^{H}=$ $\boldsymbol{Q}^{H} \boldsymbol{Q}=\boldsymbol{I}_{p}$ and $\boldsymbol{R}$ is a $p \times q$ upper triangular matrix. One method for computing the unitary matrix $Q$ is by calculating a series of Givens rotations, where each rotation will drive one of the elements beneath the diagonal of the matrix $\boldsymbol{A}$ to zero [1]. The elements of $\boldsymbol{A}$ below the diagonal are zeroed in a particular order to ensure that successive rotations do not undo previously zeroed elements. There are several different orderings that can be implemented, however for the purposes of this paper the elements are eliminated starting with the 
uppermost left element and then moving across elements beneath the diagonal in each row from left to right, before moving to the next row down. PMQRD extends this rotation matrix approach to polynomial matrices.

\section{A. Polynomial Matrix QR Decomposition}

We define the $p \times q$ complex polynomial matrix, $\underline{\boldsymbol{A}}(z)$ as a polynomial with matrix coefficients.

$$
\underline{\boldsymbol{A}}(z)=\sum_{i=t_{1}}^{t_{2}} \boldsymbol{A}_{i} z^{-i}
$$

Where $\boldsymbol{A}_{i} \in \mathbb{C}^{p \times q}$ is the $i^{t h}$ matrix tap of the polynomial matrix, $i \in \mathbb{Z}, t_{1}<t_{2}$ and $z^{-i}$ is the unit delay operator. The polynomial QR decomposition of $\underline{\boldsymbol{A}}(z)$ is shown in (4) where $\underline{\boldsymbol{Q}}(z)$ is a paraunitary polynomial matrix, so that $\underline{\boldsymbol{Q}}(z) \underline{\widetilde{\boldsymbol{Q}}}(z)=$ $\overline{\boldsymbol{I}_{p}}$ and $\underline{\boldsymbol{R}}(z)$ is an upper triangular polynomial matrix.

$$
\underline{\boldsymbol{A}}(z)=\underline{\boldsymbol{Q}}(z) \underline{\boldsymbol{R}}(z)
$$

\section{B. Elementary Polynomial Givens Rotation}

An elementary polynomial Givens rotation (EPGR) [3] is a Givens rotation preceded by an elementary time shift matrix as follows:

$$
\begin{aligned}
\underline{\boldsymbol{G}}(\alpha, \theta, \phi, t) & =\left[\begin{array}{cc}
e^{j \alpha} \cos \theta & e^{j \phi} \sin \theta \\
-e^{-j \phi} \sin \theta & e^{-j \alpha} \cos \theta
\end{array}\right]\left[\begin{array}{cc}
1 & 0 \\
0 & z^{t}
\end{array}\right] \\
& =\left[\begin{array}{cc}
e^{j \alpha} \cos \theta & z^{t} e^{j \phi} \sin \theta \\
-e^{-j \phi} \sin \theta & z^{t} e^{-j \alpha} \cos \theta
\end{array}\right]
\end{aligned}
$$

where $j$ denotes the imaginary operator and $\theta$ the rotation angle. The object of the EPGR when applied to the $2 \times 1$ polynomial vector $\boldsymbol{a}(z)$ is to drive a specific coefficient from the polynomial element $\underline{a}_{2}(z)$ to zero.

$$
\left[\begin{array}{cc}
e^{j \alpha} \cos \theta & z^{t} e^{j \phi} \sin \theta \\
-e^{-j \phi} \sin \theta & z^{t} e^{-j \alpha} \cos \theta
\end{array}\right]\left[\begin{array}{l}
\underline{a}_{1}(z) \\
\underline{a}_{2}(z)
\end{array}\right]=\left[\begin{array}{l}
\underline{a}_{1}^{\prime}(z) \\
\underline{a}_{2}^{\prime}(z)
\end{array}\right]
$$

For example, to zero the coefficient $a_{2}(i)$ then the lag parameter in the EPGR matrix is set as $t=i$ and the rotation angles are chosen [4] such that

$$
\tan \theta=\frac{\left|a_{2}(t)\right|}{\left|a_{1}(0)\right|}, \phi=-\arg \left(a_{2}(t)\right), \alpha=-\arg \left(a_{1}(0)\right)
$$

resulting in $a_{2}^{\prime}(0)=0$. The application of the EPGR renders $a_{1}^{\prime}(0)$ real. An EPGR is paraunitary by construction as each component of the matrix, i.e. the Givens rotation and the elementary time shift matrix are both paraunitary.

\section{Complete Polynomial Givens Rotation}

Successive EPGR's can be applied iteratively to the $2 \times 1$ polynomial vector $\underline{\boldsymbol{a}}(z)$ to drive all coefficients of the polynomial element $\underline{a}_{2}(z)$ arbitrarily close to zero. The coefficients of $\underline{a}_{2}(z)$ are zeroed in order of maximum magnitude. At each iteration, the coefficient of maximum amplitude is zeroed and the complete series of EPGR's required constitutes a complete polynomial Givens rotation (CPGR) [3], [4], denoted by the matrix $\underline{G}^{(2,1)}(z)$, where the superscripts denote the position of the polynomial element that the matrix is attempting to annihilate. A matrix of this form can be applied to $\underline{\boldsymbol{a}}(z)$ resulting in

$$
\underline{\boldsymbol{G}}^{(2,1)}(z)\left[\begin{array}{l}
\underline{a}_{1}(z) \\
\underline{a}_{2}(z)
\end{array}\right] \cong\left[\begin{array}{c}
\underline{a}_{1}^{\prime}(z) \\
0
\end{array}\right]
$$

\section{PMQRD Algorithm}

A CPGR can be applied to the polynomial matrix $\underline{\boldsymbol{A}}(z)$ to drive one of the polynomial elements to zero, e.g.

$$
\underline{\boldsymbol{A}}^{\prime}(z)=\underline{\boldsymbol{G}}^{(j, k)}(z) \underline{\boldsymbol{A}}(z)
$$

where $\underline{G}^{(j, k)}(z)$ is the CPGR matrix designed to drive all coefficients of $\underline{a}_{j, k}(z)$ sufficiently small, resulting in a new polynomial matrix, $\underline{\boldsymbol{A}}^{\prime}(z)$ [3], [4]. The CPGR applies an iterative sequence of EPGR matrices. Each EPGR is formulated as a $p \times p$ identity matrix with the exception of the four elements positioned at the intersection of rows $j$ and $k$ with columns $j$ and $k$. These elements are given by the $2 \times 2$ sub-matrix, $\underline{\boldsymbol{G}}(\alpha, \theta, \phi, t)$ as in (6), where for example if the dominant coefficient is $a_{j, k}(i)$, then the lag parameter is set as $t=i$ and the coefficients required for calculating the rotation angles are $a_{2}(t)=a_{j k}(i)$ and $a_{1}(0)=a_{k, k}(0)$.

Zeroing the polynomial elements of $\underline{\boldsymbol{A}}(z)$ beneath the diagonal in the order previously described is now a simple case of applying successive CPGR matrices. Defining the paraunitary matrix $\underline{\boldsymbol{Q}}_{\beta}(z)$ as:

$$
\underline{\widetilde{\boldsymbol{Q}}}_{\beta}(z)=\underline{\boldsymbol{G}}_{\beta}(z) \ldots \underline{\boldsymbol{G}}_{2}(z) \underline{\boldsymbol{G}}_{1}(z)
$$

where $\beta$ represents an unspecified number of iterations. After $\beta$ iterations (9) results in:

$$
\underline{\boldsymbol{R}}(z)=\underline{\widetilde{\boldsymbol{Q}}}_{\beta}(z) \underline{\boldsymbol{A}}(z)
$$

\section{APPlication of PMQRD to MIMO ChANNEL EQUALIZATION}

Without loss of generality we consider a frequency selective MIMO system of equal number transmit and receive antennas, i.e. $M_{r}=M_{t}$. The PMQRD of the channel $\underline{\boldsymbol{H}}(z)$ is shown in (12).

$$
\underline{\boldsymbol{H}}(z)=\underline{\boldsymbol{Q}}(z) \underline{\boldsymbol{R}}(z)
$$

A set of source signals of length $N, \boldsymbol{s}(n) \in \mathbb{C}^{M_{t} \times 1}$ for $n \in$ $\{0,1, \ldots, N-1\}$ are propagated through the MIMO wireless channel, $\underline{\boldsymbol{H}}(z)$, received and filtered with $\widetilde{\boldsymbol{Q}}(z)$, as shown in Figure 1 .

$$
\boldsymbol{y}=\underline{\widetilde{\boldsymbol{Q}}}(z) \underline{\boldsymbol{H}}(z) \boldsymbol{s}+\underline{\widetilde{\boldsymbol{Q}}}(z) \boldsymbol{n}
$$

where $\boldsymbol{n}$ denotes an additive Gaussian noise process with variance $\sigma^{2} \boldsymbol{I}_{M_{r}}$. The convolutive mixing model can be rewritten as (14), where $\boldsymbol{n}^{\prime}=\underline{\widetilde{Q}}(z) \boldsymbol{n}$.

$$
\boldsymbol{y}=\underline{\boldsymbol{R}}(z) \boldsymbol{s}+\boldsymbol{n}^{\prime}
$$




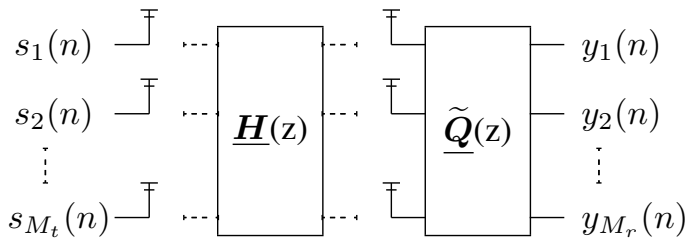

Fig. 1. PMQRD system diagram

\section{A. Iterative Interference Cancellation}

The MIMO channel problem can now be transformed into a set of $M_{r}$ equalization problems using back substitution. The $M_{t}^{t h}$ source signal is expressed as (15) which is a single channel equalization problem, which is solved using a minimum mean squared error (MMSE) equalizer [5].

$$
\boldsymbol{y}_{M_{r}}=\underline{\boldsymbol{r}}_{M_{r} M_{r}}(z) \boldsymbol{s}_{M_{t}}+\boldsymbol{n}_{M_{r}}^{\prime}
$$

Once $s_{M_{r}}$ is retrieved we use it to cancel its contribution to $\boldsymbol{y}_{M_{r}-1}$ as follows

$$
\boldsymbol{y}_{M_{r}-1}-\underline{\boldsymbol{r}}_{M_{r}-1 M_{r}}(z) \boldsymbol{s}_{M_{t}}=\underline{\boldsymbol{r}}_{M_{r}-1 M_{r}-1}(z) \boldsymbol{s}_{M_{t}-1}+\boldsymbol{n}_{M_{r}-1}^{\prime}
$$

which again is a single channel equalization problem. Therefore the $i^{\text {th }}$ single channel equalization problem can be formulated as

$$
\boldsymbol{y}_{i}-\sum_{j=i+1}^{M_{r}} \underline{\boldsymbol{r}}_{i j}(z) \boldsymbol{s}_{j}=\underline{\boldsymbol{r}}_{i i}(z) \boldsymbol{s}_{i}+\boldsymbol{n}_{i}^{\prime}
$$

providing the streams $s_{i+1} \ldots s_{M_{t}}$ have been previously recovered.

\section{Channel Model}

In our simulations, we consider a MIMO system with three antennas at the transmitter and receiver. The temporal length, $L$ of the channel between each transmitter and receiver is five. The channel has a constant power delay profile with equal average gain for each tap.

\section{Horizontal EnCODing (H-BLAST)}

H-BLAST [6] is a sub-optimal encoding architecture used to simplify receiver design and attains a maximum diversity order of $M_{r}$ as any given symbol is transmitted from only one transmit antenna and received by $M_{r}$ antennas [2]. The transmitter architecture is shown in Figure 2.

The data stream is first demultiplexed into three substreams, $s_{1}, s_{2}, s_{3}$. Each substream is then independently convolutionally encoded, interleaved and symbol mapped prior to transmission. We have used the code formatting polynomials in (18) as per the global system for mobile (GSM) CS1-CS3 [7].

$$
\begin{aligned}
& G 0=1+D^{3}+D^{4} \\
& G 1=1+D^{1}+D^{3}+D^{4}
\end{aligned}
$$

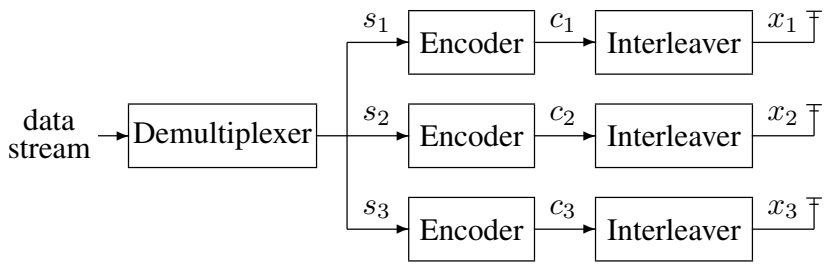

Fig. 2. H-BLAST transmitter architecture

To ensure that errors appear random and to avoid long error bursts, an interleaver is used to randomize the encoded bits prior to transmission. We have used an S-Random interleaver with a depth of 28 bits to gain maximum performance [8].

\section{A. H-BLAST Receiver Design}

The received signals are filtered with $\underline{\widetilde{Q}}(z)$ as shown in Figure 1, yielding the received substreams, $y_{1}, y_{2}, y_{3}$. Each substream is turbo equalized prior to the application of the iterative interference cancellation scheme previously described.

\section{B. Turbo Equalizer}

Turbo equalization [9] is an iterative equalization and decoding technique that can achieve impressive performance gains for coded data transmission over intersymbol interference (ISI) channels. Repeating the equalization and decoding tasks on the same set of data and incorporating soft feedback from the decoder into the equalization process generally yields significant improvements in the BER [9]. We assume the channel coefficients of the $i^{t h}$ stream, $\underline{\boldsymbol{r}}_{i i}(z)$ are known to the receiver and do not vary in time within each block. Figure 3 shows the turbo equalization structure.

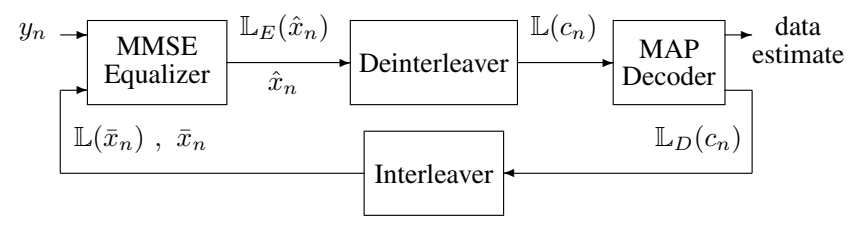

Fig. 3. Turbo equalization structure

The $\mathbb{L}$ operator is applied to quantities $x \in\{+1,-1\}$ and is given by

$$
\mathbb{L}(x)=\ln (P(x=+1) / P(x=-1))
$$

i.e., the log likelihood ratio (LLR).

\section{MMSE Equalizer}

The MMSE equalizer computes estimates $\hat{x}_{n}$ of the transmitted symbols $x_{n}$ from the received symbols $y_{n}$ by minimizing the cost function $E\left\{\left|x_{n}-\hat{x}_{n}\right|^{2}\right\}$ [5] where $\hat{x}_{n}$ represents the soft output from the MMSE equalizer, and $E\{\cdot\}$ denotes the statistical expectation operator.

For a single channel $\underline{\boldsymbol{r}}_{i i}(z)$ the channel is denoted as $h_{0}+h_{1} z^{-1}, \ldots, h_{L-1} z^{-(L-1)}$ where $L$ represents the channel length. We only consider taps of $\underline{\boldsymbol{r}}_{i i}(z)$ that are greater than 
$\sigma^{2} /\left(M_{r} M_{t}\right)$ where $\sigma^{2}$ represents the noise variance. We set the length of the equalizer, $M$, to capture these taps of interest. The channel convolution matrix, $\boldsymbol{H} c$ of dimensions $M \times(M+L-1)$ takes the form:

$$
\boldsymbol{H} c=\left[\begin{array}{ccccccc}
h_{0} & h_{1} & \ldots & h_{L-1} & 0 & \ldots & 0 \\
0 & h_{0} & h_{1} & \ldots & h_{L-1} & \ldots & 0 \\
& & & \ddots & \ddots & \ddots & \ddots \\
0 & \ldots & 0 & h_{0} & h_{1} & \ldots & h_{L-1}
\end{array}\right]
$$

Let $\boldsymbol{H} c_{M}$ denote the column of $\boldsymbol{H} c$ containing the most energy. Assuming symbols are temporally uncorrelated, we write $E\left\{\left(\boldsymbol{x}_{n}-\overline{\boldsymbol{x}}_{n}\right)\left(\boldsymbol{x}_{n}-\overline{\boldsymbol{x}}_{n}\right)^{H}\right\}$ as a diagonal matrix $\operatorname{diag}(\boldsymbol{v})$ where the $n^{t h}$ element of $\boldsymbol{v}$ is $\boldsymbol{v}_{n}=1-\bar{x}_{n}^{2}$ and $\bar{x}_{n}$ denotes the interleaved soft estimates of the transmitted symbol from the MAP decoder output. The MMSE weight vector, $\boldsymbol{w}_{n}$ is given by [9] [5]:

$$
\boldsymbol{w}_{n}=\left(\boldsymbol{H} \boldsymbol{c} \times \operatorname{diag}\left(\boldsymbol{v}_{n}\right) \times \boldsymbol{H} c^{H}+\sigma^{2} \boldsymbol{I}\right)^{-1} \boldsymbol{H} c_{M}
$$

The MMSE equalizer output $\hat{x}_{n}$ is used to obtain the difference between the posteriori and a prior LLR as follows:

$$
\begin{aligned}
\mathbb{L}_{E}\left(\hat{x}_{n}\right) & =\ln \frac{p\left\{x_{n}=+\left.1\right|_{\hat{x}_{n}}\right\}}{p\left\{x_{n}=-\left.1\right|_{\hat{x}_{n}}\right\}}-\ln \frac{p\left\{x_{n}=+1\right\}}{p\left\{x_{n}=-1\right\}} \\
& =\ln \frac{p\left\{\left.\hat{x}_{n}\right|_{s_{n}=+1}\right\}}{p\left\{\left.\hat{x}_{n}\right|_{s_{n}=-1}\right\}} \\
& =\frac{4 \Re\left\{\hat{x}_{n}\right\}}{1-\boldsymbol{H} c_{M}^{T} \boldsymbol{w}_{n}}
\end{aligned}
$$

where $\Re\left\{\hat{x}_{n}\right\}$ denotes the real component of the quantity $\left\{\hat{x}_{n}\right\}$.

\section{MAP Decoder}

The maximum a posteriori (MAP) algorithm [10] computes the posterior probability of symbols from Markov sources transmitted through discrete memoryless channels. Since the output of a convolutional coder passed through the equalized frequency selective channel forms a Markov source the MAP algorithm can be used for maximum a posteriori probability decoding of convolutionaly encoded code [11]. For each transmitted symbol it generates a hard estimate (using thresholding) and soft outputs, $\mathbb{L}_{D}\left(c_{n}\right), \bar{x}_{n}$ in the form of the a posteriori probability of the received sequence [12].

\section{E. Optimal Detection Ordering}

The performance of the iterative interference cancellation scheme is affected by the order in which the components of $\boldsymbol{x}$ are detected. An optimal detection ordering (ODO) scheme can significantly improve system performance. This is achieved by swapping the columns of $\underline{\boldsymbol{H}}(z)$ and performing the PMQRD. A permutation of the columns of $\underline{\boldsymbol{H}}(z)$ exists such that $\left\|\boldsymbol{r}_{3,3}\right\|_{F}$ is maximal. Wolniansky et al have shown [13] that the column permutation of $\underline{\boldsymbol{H}}(z)$ where maximising $\left\|\boldsymbol{r}_{i+1, i+1}\right\|_{F}$ given that $\left\|\boldsymbol{r}_{i, i}\right\|_{F}$ is already maximal yields the order of optimum detection. After all three streams have been recovered in the receiver, the inverse of this permutation is applied to match the decoded stream from each receiver antenna with the stream from the corresponding transmit antenna.

\section{VERTICAL ENCODING (V-BLAST)}

A Vertical BLAST (V-BLAST) architecture is shown in Figure 4 (we follow the convention in [2]). The data stream undergoes convolutional encoding, interleaving and symbol mapping prior to demultiplexing into $M_{t}$ sub-streams. This form of encoding is potentially optimal since each information bit is spread across multiple antennas. However receiver complexity is increased as V-BLAST requires joint decoding of the sub-streams.

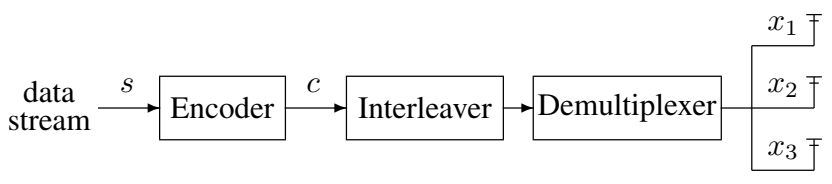

Fig. 4. V-BLAST transmitter architecture

Again we have used the code formatting polynomials in (18). Due to the increased bit-length of the encoded stream, $c$ interleaver depth has been increased to 55 bits.

Due to the benefit in increased diversity order the additional performance benefit from implementing an ODO scheme is negligible for a V-BLAST system versus the increase in computational complexity in the receiver. Hence an ODO VBLAST scheme has not been implemented.

\section{A. V-BLAST Receiver Design}

As the substreams can no longer be independently MAP decoded, initially the soft estimate output from the MMSE equalizer is quantized to the BPSK modulation scheme used in the transmitter and used for iterative interference cancellation. Once all the substreams have been recovered, the soft estimates are multiplexed, deinterleaved and MAP decoded. The hard decoded MAP estimate of $\boldsymbol{x}$ is then used for iterative interference cancellation on subsequent iterations of the turbo equalization loop while the soft estimates are used for MMSE equalization.

\section{RESULTS}

We consider a wide sense stationary (WSS) situation where the channel coefficients have been assumed to be unchanged within each data block, but allowed to change between data blocks according to a zero mean complex circular Gaussian distribution. WSS implies that the second-order time statistics of the channel are stationary and is justified in mobile channels over short periods [2]. The bit error rate has been computed for 1000 Monte Carlo simulations. The modulation scheme used is BPSK for evaluation purposes but extension to large constellations is straightforward. The number of time slots of the channel, $N=2048$. We assume that the receiver has perfect channel knowledge. 


\section{A. H-BLAST}

We have used a MIMO-OFDM QR scheme as a benchmark. MIMO-OFDM is a DFT based technique that decomposes the otherwise frequency selective channel of bandwidth $B$ into $N$ orthogonal frequency flat MIMO channel, each with a bandwidth $B / N$ [2]. The data stream undergoes the same encoding process as the PMQRD based scheme. Prior to transmission the transmitter performs an inverse fast Fourier transform (IFFT) operation on the signal to be transmitted from each individual transmit antenna. A cyclic prefix (CP) of length (L-1) is then added prior to transmission. At the receiver the cyclic prefix is stripped off and an FFT is applied to the signal received at each antenna. The standard $\mathrm{QR}$ decomposition is then applied within each narrowband tone. The iterative cancellation within the receiver is performed on each tone individually and finally a Viterbi decoder is used to remove the error correction coding. OFDM transmission incurs on average a loss in spectral efficiency of $(L-1) /(N+L-1)$ on account of the cyclic prefix. If $N \gg L$, this loss is negligible [2] so this has not been considered.

Figure 5 directly compares the proposed PMQRD and MIMO-OFDM QR schemes for both standard and ODO implementations. BER performance of the PMQRD is far superior to the MIMO-OFDM QR scheme, for example a $2 \mathrm{~dB}$ gain in SNR is observed at BER $10^{-3}$ when ODO is applied.

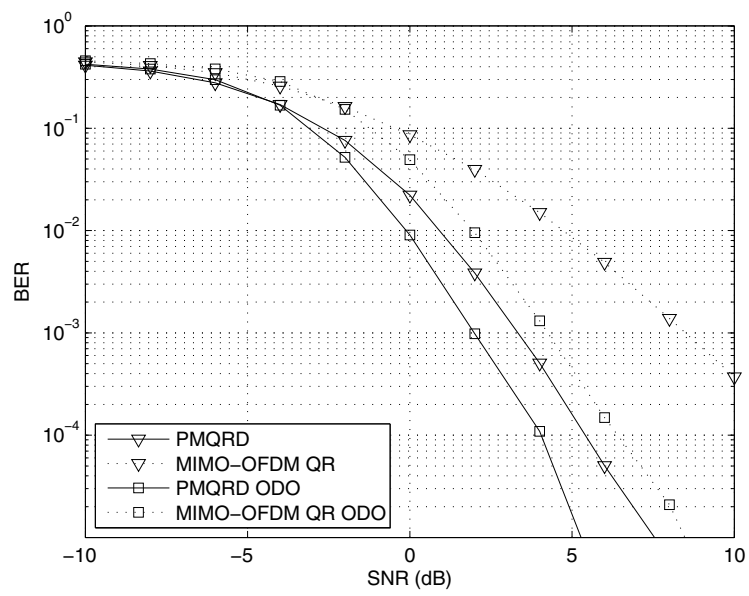

Fig. 5. Average uncoded BER results for H-BLAST PMQRD and MIMOOFDM schemes for a $3 \times 3$ MIMO channel, $L=5$, with constant power profile

\section{B. V-BLAST}

Figure 6 shows the superior performance of the V-BLAST scheme when compared to H-BLAST due to the increased diversity gain. Again the benefit of PMQRD over the MIMOOFDM approach is clearly shown, with a $5 \mathrm{~dB}$ gain in SNR at BER $10^{-4}$ for the V-BLAST schemes.

\section{CONCLUSION}

We have proposed a PMQRD technique for MIMO systems with frequency selective channels and implemented a BLAST architecture based communications system.

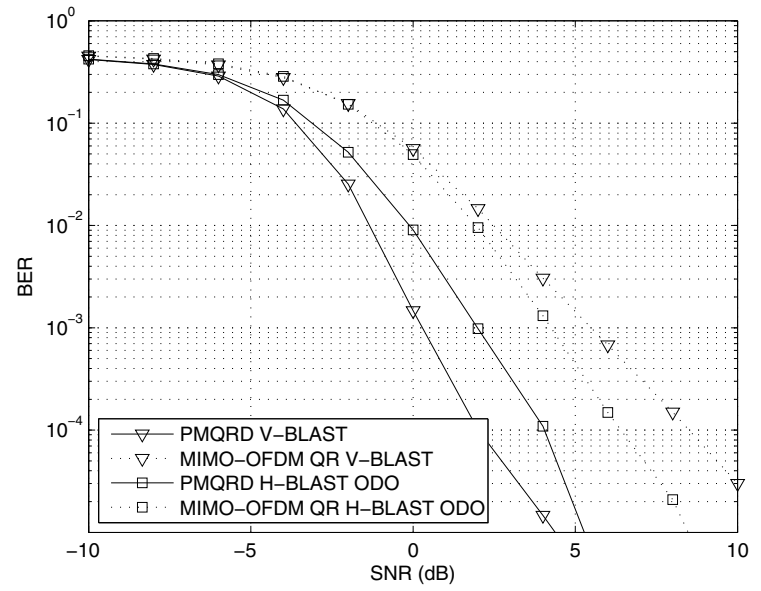

Fig. 6. Average uncoded BER results for V-BLAST and H-BLAST PMQRD and MIMO-OFDM schemes for a $3 \times 3$ MIMO channel, $\mathrm{L}=5$, with constant power profile

For the MIMO-OFDM BLAST schemes the information in each individual bit is constrained to a single narrowband tone. Individual tones may have poor gain due to the frequency selective nature of the MIMO channel, resulting in degraded system performance. However in the PMQRD BLAST based system the information in each bit is spread across the entire frequency bandwidth, making the system more robust to frequency selectivity, resulting in superior performance. This makes PMQRD highly suitable for MIMO-QR based applications where the transmitter has no prior channel knowledge, for example digital video broadcasting.

For modulation schemes with constant transmit energy per symbol, the peak-to-average power ratio (PAR) will be unity for PMQRD based schemes. For an identical OFDM based scheme, the IFFT operation at each transmit antenna results in a relatively high PAR. Therefore, nonlinearities may get overloaded by high signal peaks, causing intermodulation distortion in the transmitted signal [14], and undesired outof-band radiation. If radio frequency (RF) power amplifiers are operated without large power back-offs, it is impossible to keep the out-of-band power below specified limits, leading to very inefficient amplification and expensive transmitters [15]. For modulation schemes with variable transmit energy per symbol, the PAR of a PMQRD system will still be significantly less than that of OFDM, reducing transmitter complexity and cost.

\section{ACKNOWLEDGMENT}

The authors would like to thank Dr Ian Prouder for his invaluable comments and suggestions. The work of Martin Davies is jointly funded by EPSRC and QinetiQ.

\section{REFERENCES}

[1] G. Golub and C. Loan, Matrix Computations, 3rd ed. Baltimore, Maryland: The John Hopkins University Press, 1996. 
[2] A. Paulraj, R. Nabar, and D. Gore, Introduction to Space-Time Wireless Communications, 1st ed. University Press, Cambridge: Cambridge University Press, 2003.

[3] J. Foster, J. McWhirter, and J. Chambers, "An algorithm for computing the QR decomposition of a polynomial matrix," in $15^{\text {th }}$ International Conference on Digital Signal Processing, Cardiff, July 2007.

[4] - "A polynomial matrix QR decomposition with application to MIMO channel equalization," in Conference Digest : $41^{\text {st }}$ Asilomar conference on signals, systems, and computers, November 2007.

[5] M. Tuchler, R. Koetter, and A. Singer, "Turbo equalization: principles and new results," IEEE transactions on communications, vol. 50, no. 5, pp. 754-767, May 2002.

[6] G. Foschini, "Layered space-time architecture for wireless communication in a fading environment when using multi-element antennas," Bell Labs Technical Journal (Autumn), pp. 41-59, 1996.

[7] Technical Specification Group GERAN, Channel Coding, 3rd Generation Partnership Project Std. 3GPP TS 05.03 V8.6.1 (2001-01), 1999.

[8] M. Valenti. (2006) Coded modulation library. [Online]. Available: http://www.iterativesolutions.com/Matlab.htm
[9] R. Koetter, A. Singer, and M. Tuchler, "Turbo equalization: an iterative equalization and decoding technique for coded data transmission," IEEE Signal Processing Magazine, pp. 67-80, January 2004.

[10] L. Bahl, J. Cocke, F. Jelinek, and J. Raviv, "Optimal decoding of linear codes for minimizing symbol error rate," IEEE Transactions on Information Theory, vol. 20, pp. 284-287, March 1974.

[11] T. Moon, Error Correction Coding: Mathematical Methods and Algorithms, 1st ed. Hoboken, New Jersey: John Wiley \& Sons, 2005.

[12] B. Vucetic and J. Yuah, Turbo Codes: Principles and Applications, 1st ed. Dordrecht, The Netherlands: Kluwer Academic Publishers, 2000.

[13] P. Wolninansky, G. Foschini, G. Golden, and R. Valenzuela, "V-BLAST: An architecture for realizing very high data rates over the rich-scattering wireless channel," in 1998 International Symposium on Signals, Systems and Electronics, Pisa, Sept-Oct 1998, pp. 295-300.

[14] J. Proakis, Digital Communications, 4th ed. Avenue of the Americas, New York: McGraw-Hill, 2001.

[15] S. Muller and J. Huber, "A comparison of peak power reduction schemes for OFDM," in Proceedings of IEEE GLOBECOM, Phoenix, Arizona, November 1997, pp. 1-5. 